تأثير تشميس التربة والفطر Trichoderma harzianum في مكافحة مرض الذبور فيول الفيوزاريومي ونيماتودا تعقد جذور البطيخ الأصفر في ظروف محافظة دير الزور (سورية)

\author{
فيصل المظهور \\ حسين الاخيل \\ قسم وقاية النبات، كلية الزر اعة، جامعة الفرات، سوريا
}

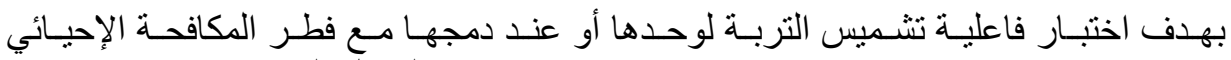

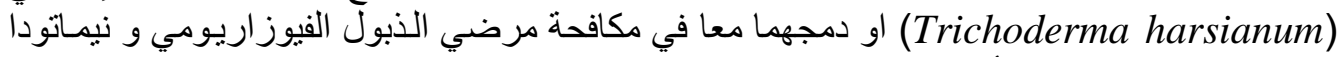

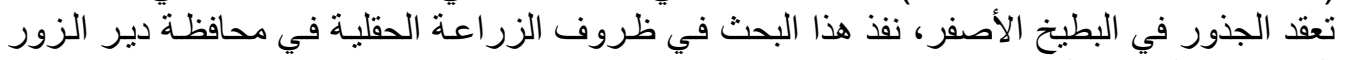
الواقعة في المنطقة الثرقية من سورية.

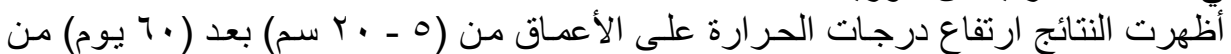

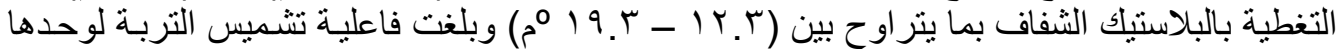

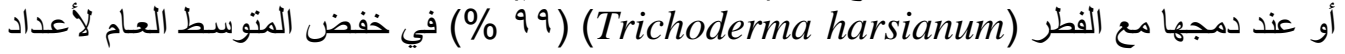

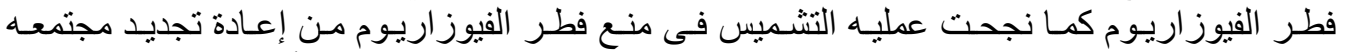

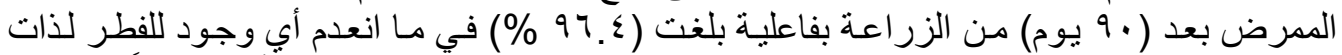

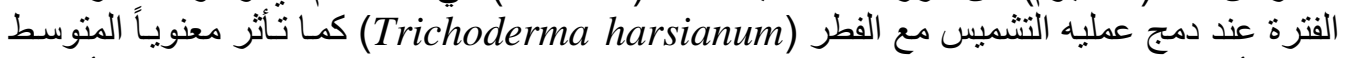

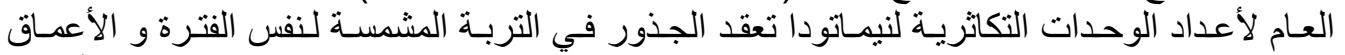

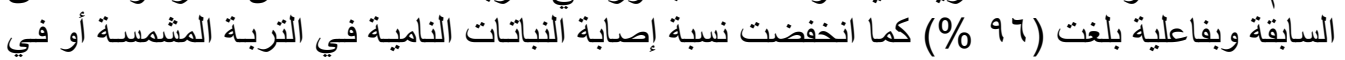

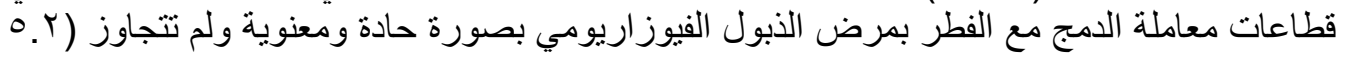

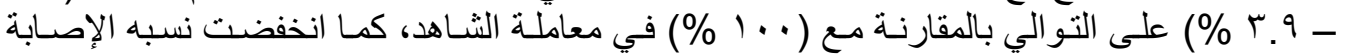

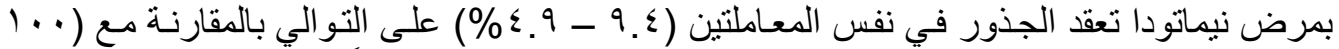

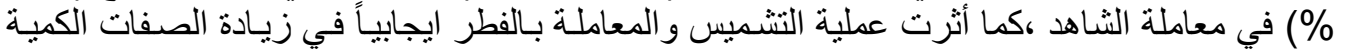

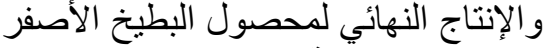
الكلمات المفتاحية : تشميس التربة، الفطر الطفيلي ( T. harzianum)، نبماتودا تعقد الجذور، الذبول الفيوز اريومي، البطيخ الأصفر.

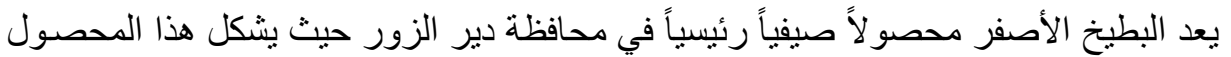

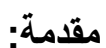

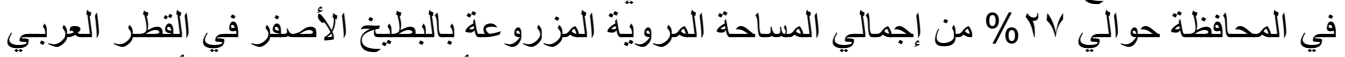

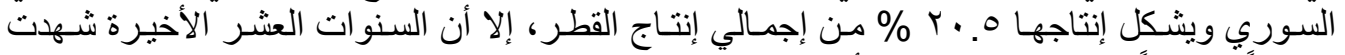

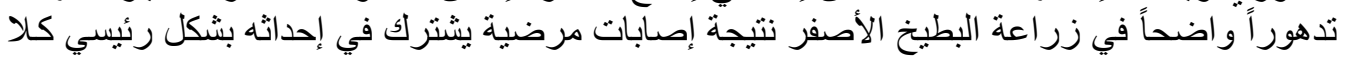

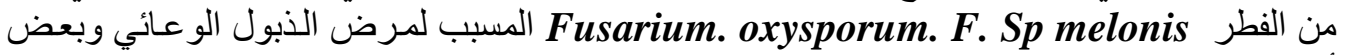

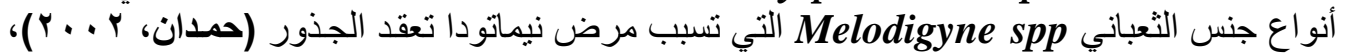

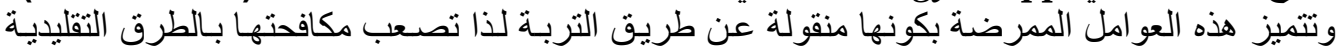

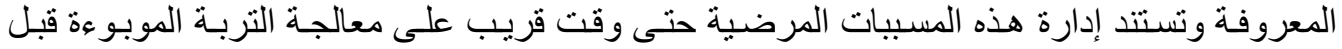

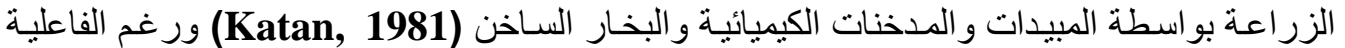

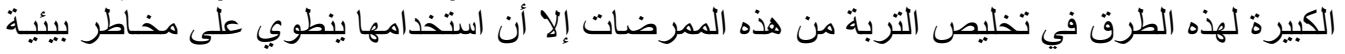

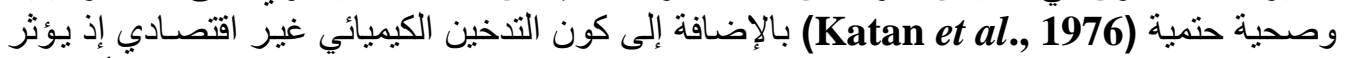

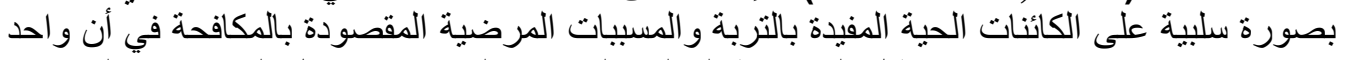

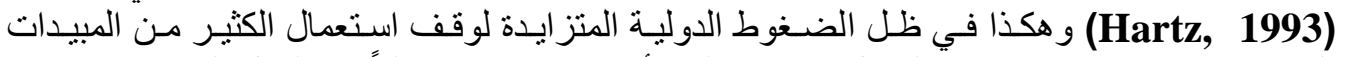

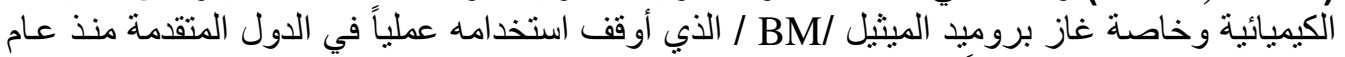

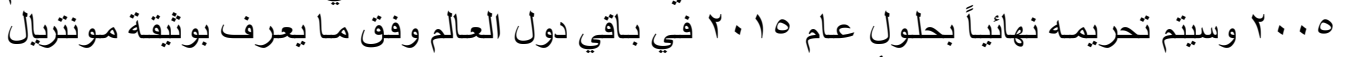
وبما أن القطر العربي السوري من الدول المرشحة لتطبيق هذه الاتفاقيـة

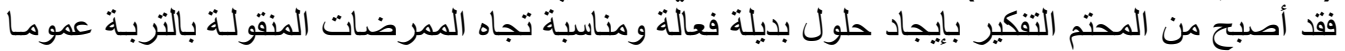

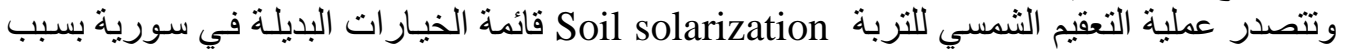

Fayoum J. Agric. Res. \& Dev., Vol.23, No.1, January, 2009 
$0 \wedge$

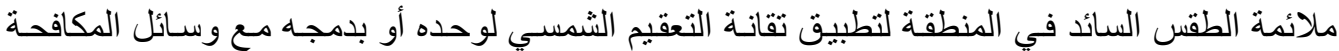
الكيميائية أو الحيوية في إطار المكافحة المتكاملة للآفات الزر اعية / IBM / ولذلك تهذف هذه الدر اسـة

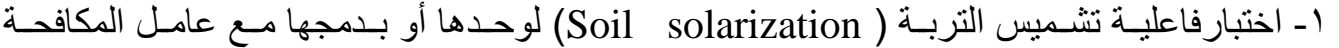

F. Trichoderma harzianum الإحيائيـة الفطر الطفيليلي

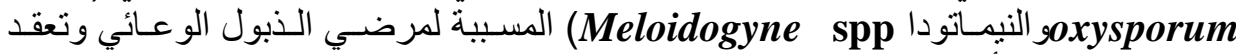

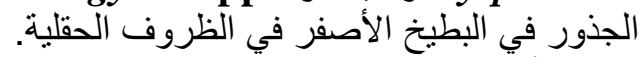

r- در اسة نأثتير تشميس التربة وعو امل المكافحة الإحيائية الدذكورة في غلة البطيخ الأصفر وبعض مكوناتها.

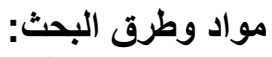

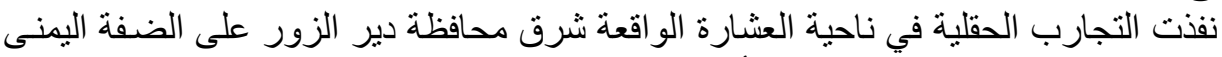

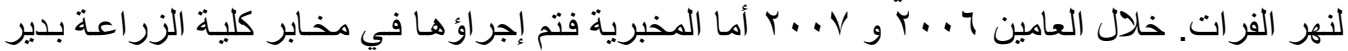

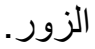

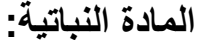

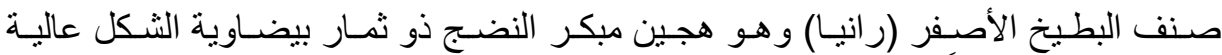

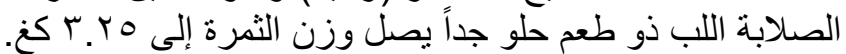

عو امل المكافحة الإحيائية: Trichoderma harzianum الفط الفئة

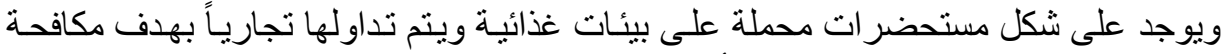

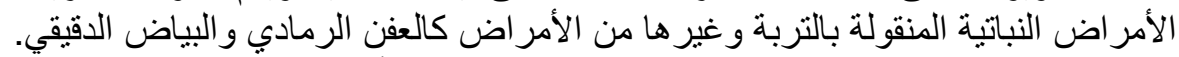

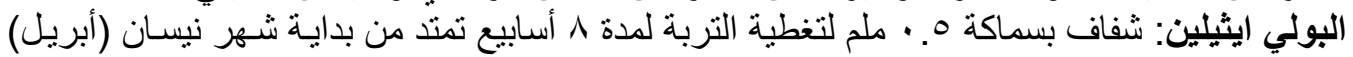

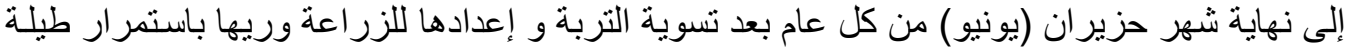
فترة التشميس.

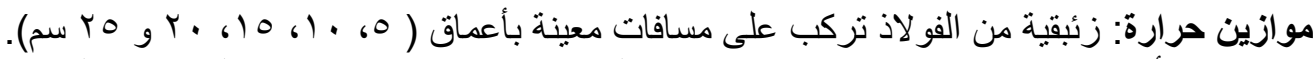

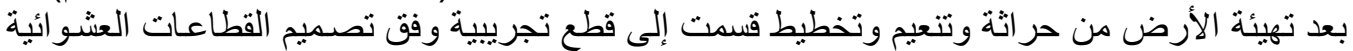

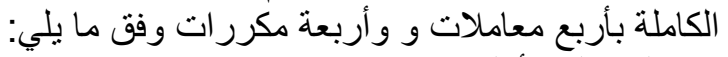

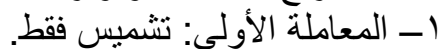

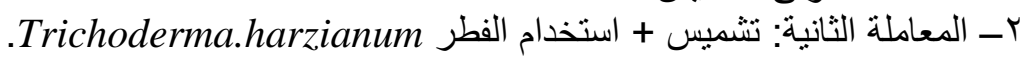

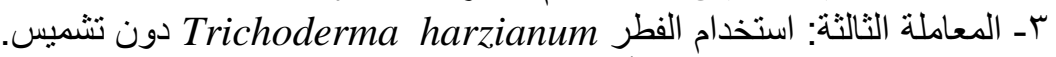

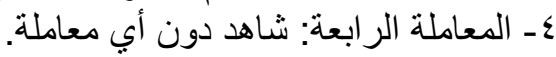

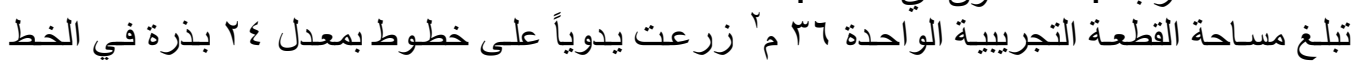

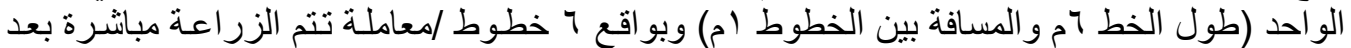

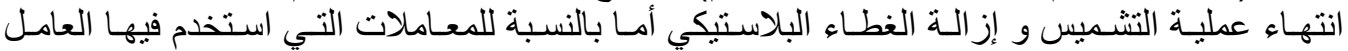

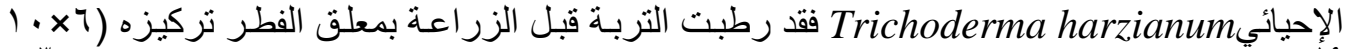

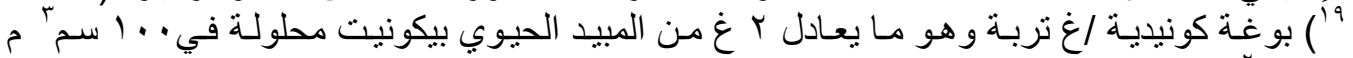

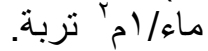

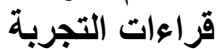

ا- - إجر اء تحليل ميكانيكي وكيميائي لتربة الحقل قبل عملية التشميس وبعدها مباشرة.

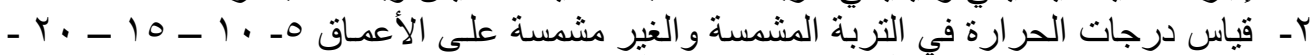
ro

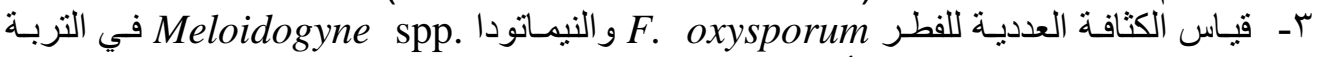

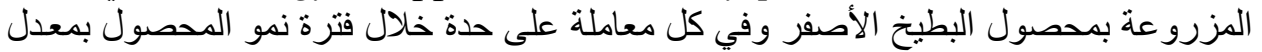

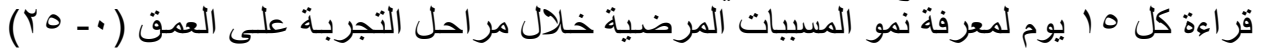

Fayoum J. Agric. Res. \& Dev., Vol.23, No.1, January, 2009 
ع - ت تقييم حالات الذبول أثناء فصل النمو وبمعدل 10 يوم بين القر اءة و الأخرى وفي نهايـة نمو

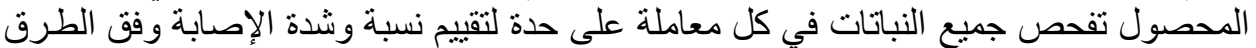

المذكورة في الفقرات السابقة. 0ـ قياس طول النبات (سم) في نهاية موسم النمو.

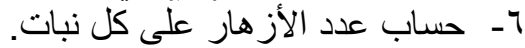

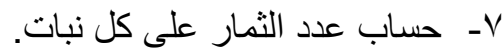

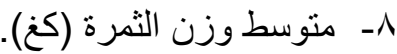

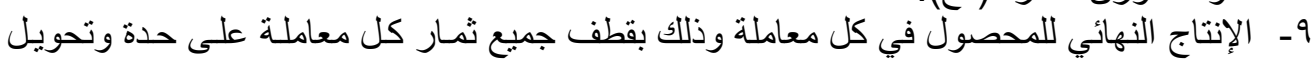

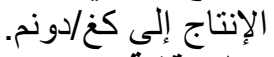

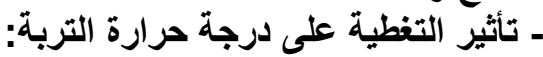

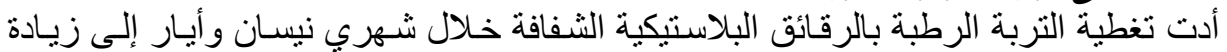

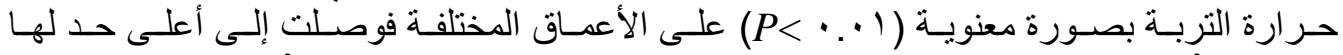

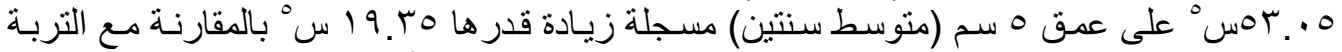

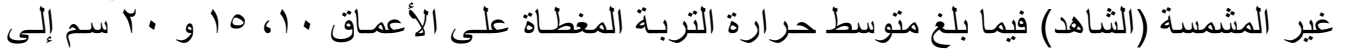

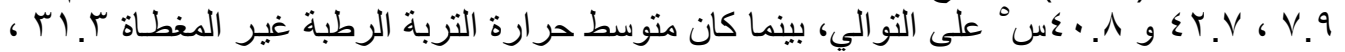
1.

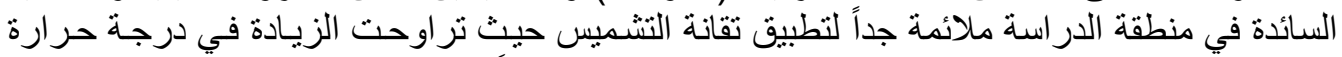

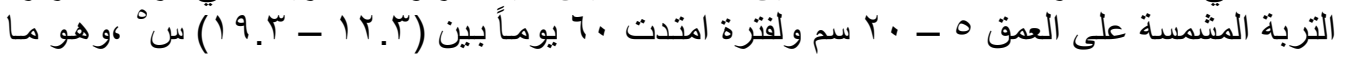

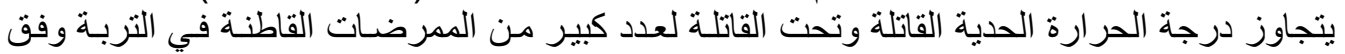

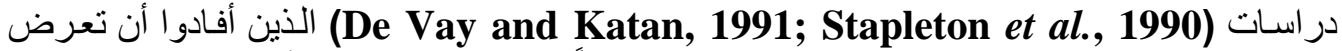

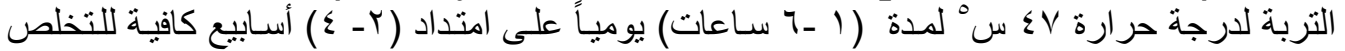

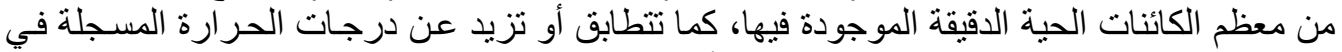

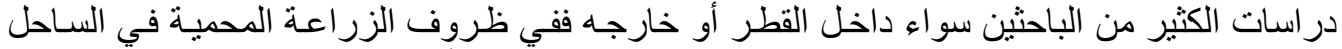

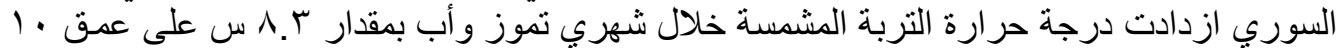

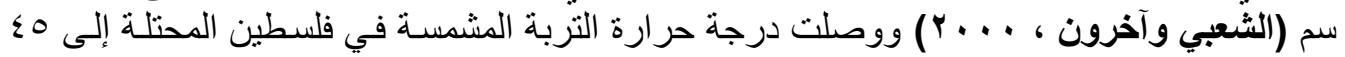

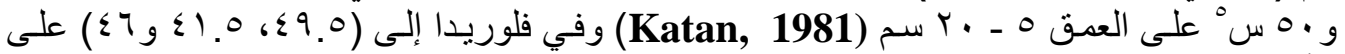

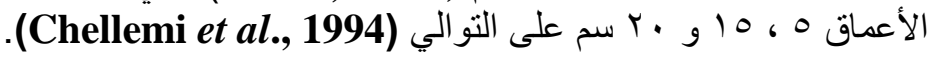

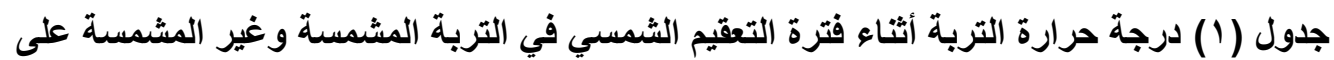

\begin{tabular}{|c|c|c|c|}
\hline مقدار الزيادة & تربة مشمسة & الثـاهد & عمق التربة (سم) \\
\hline 9.1 & $7 . r$ & 0.0 & السطح \\
\hline $19 . r$ & or.o & rr.v & 0 \\
\hline 17.7 & $\varepsilon V .9$ & $\Gamma .1$ & 1. \\
\hline 10.7 & $\varepsilon \varepsilon V$ & $r q .1$ & 10 \\
\hline IT.r & $\varepsilon \cdot . \wedge$ & $r \wedge .0$ & $r$. \\
\hline
\end{tabular}

ـ ت تأثير المعاملات على تعداد الفظر والنيماتودا:

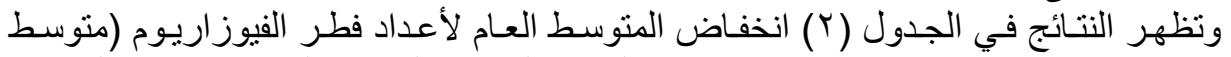

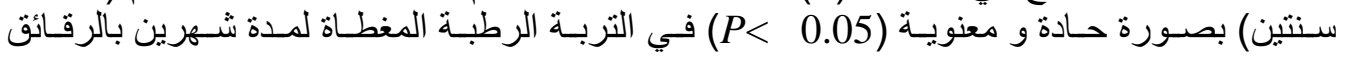

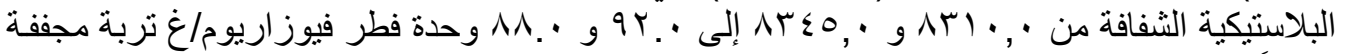

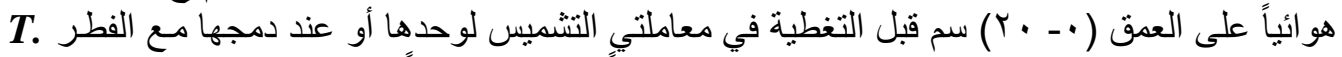

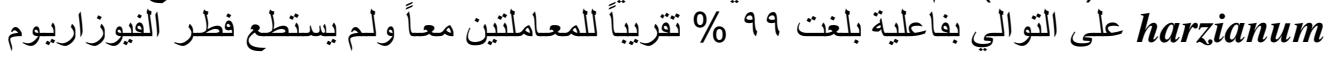

Fayoum J. Agric. Res. \& Dev., Vol.23, No.1, January, 2009 
7.

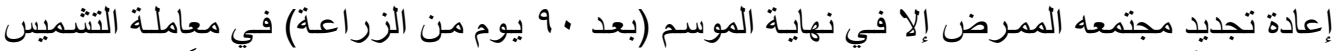

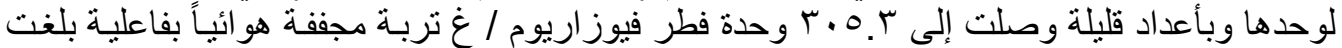

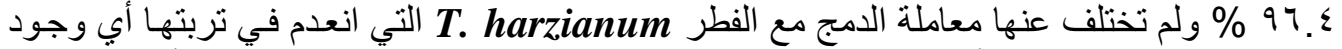

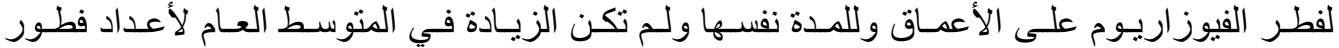

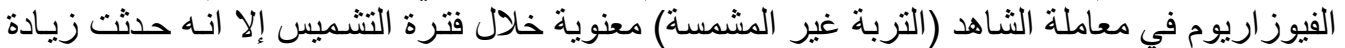

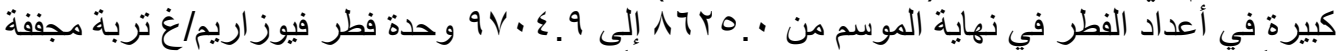

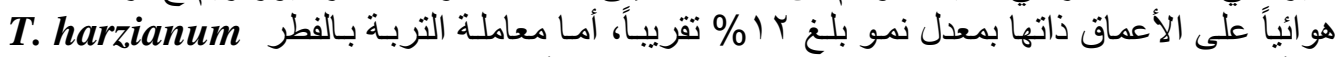

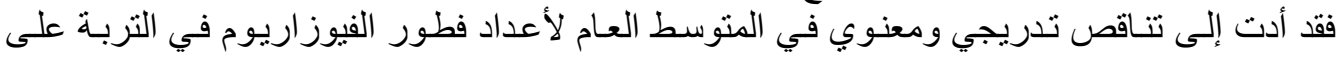

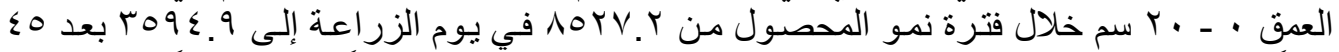

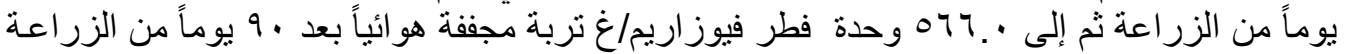

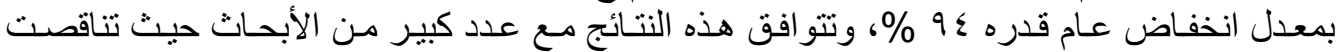

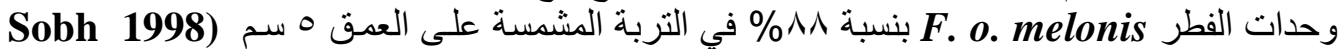
(and Abou-jawdah,

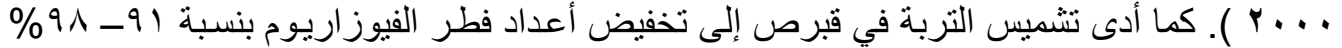

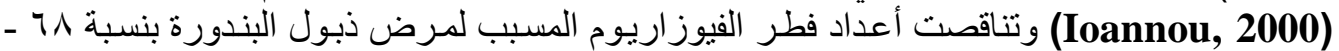

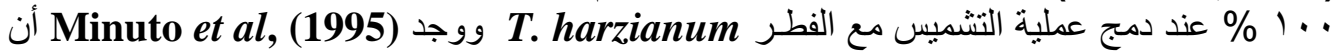

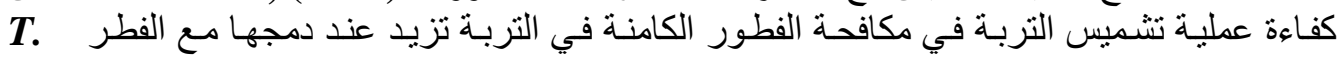

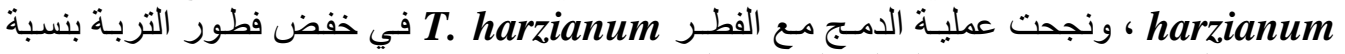

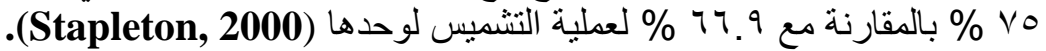

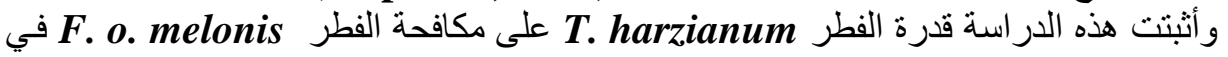

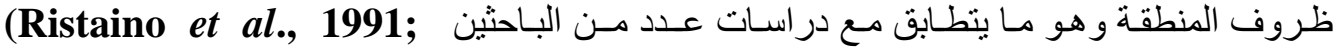
Harman et al., 1993; Green, 1980 ) جدول (Y) تأثير عملية التشميس وإضافة الفطر T. harzianum في كثافة فطور الفيوزاريوم في

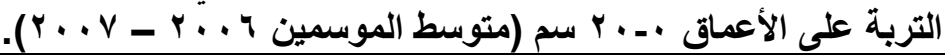

\begin{tabular}{|c|c|c|c|c|}
\hline \multicolumn{4}{|c|}{ متوسط عدد وحدات الفيوزاريوم/ غ تربة مجففة هوائيا } & \multirow{3}{*}{ موعد القراعة المعلات } \\
\hline \multirow{2}{*}{ بعد · 9 يوم من } & \multirow{2}{*}{ بعد مث الزراعةم } & \multirow{2}{*}{ التشميس } & \multirow{2}{*}{ 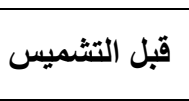 } & \\
\hline & & & & \\
\hline $9 V \cdot\{.9$ & $q \pi \leqslant 0$. & NTro." & ATrO.. & الثـاهد \\
\hline$r .0 . r$ & $191 . \cdot$ & 94 & $\Delta r \cdot \cdot \cdot \cdot$ & التشميس \\
\hline 074. & $r \Delta q \varepsilon . q$ & AOYV.r & - & T harzianum الفطر \\
\hline - & $\leq 7$. & $\Lambda \wedge . Y$ & $A r \leqslant 0 . \cdot$ & T.harzianum تشميس + الفطر \\
\hline \multicolumn{3}{|c|}{$r r v .7$} & بين المعاملات & \multirow{2}{*}{ LSD $_{0.05}$} \\
\hline \multicolumn{3}{|c|}{ ro.. } & بين القراءات & \\
\hline
\end{tabular}

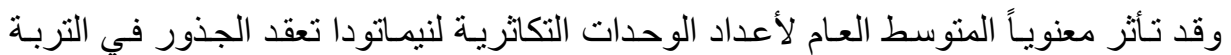

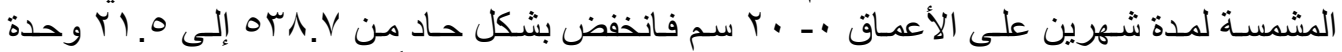

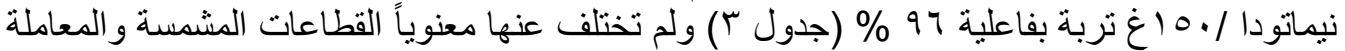

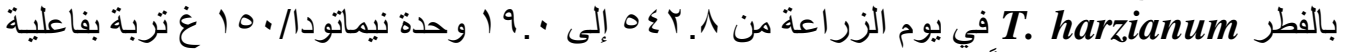

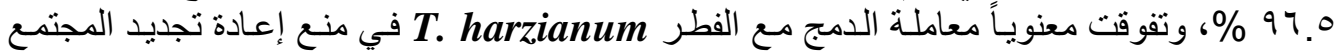

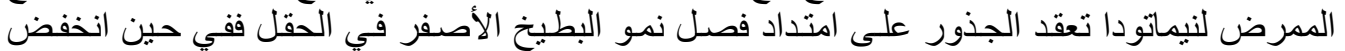

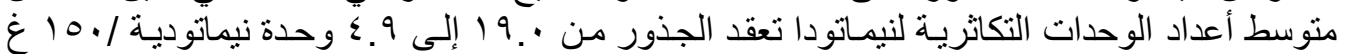

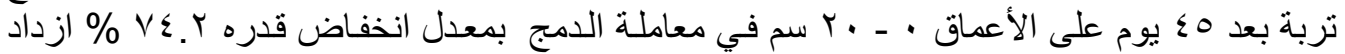

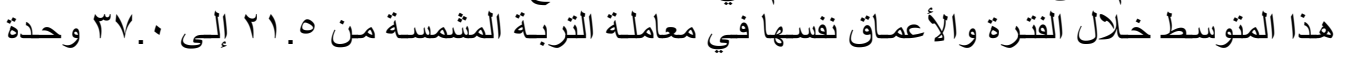

Fayoum J. Agric. Res. \& Dev., Vol.23, No.1, January, 2009 


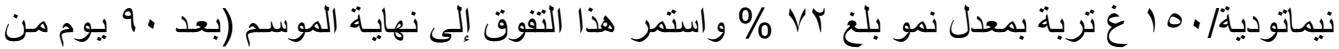

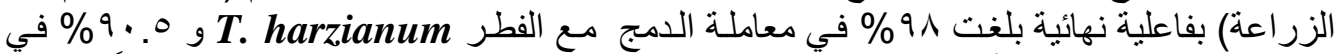

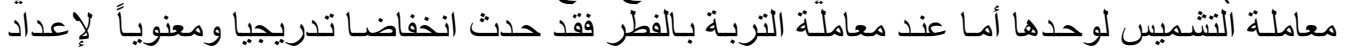

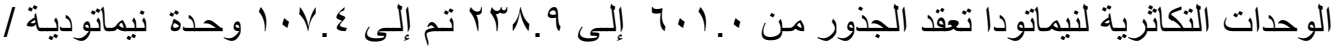

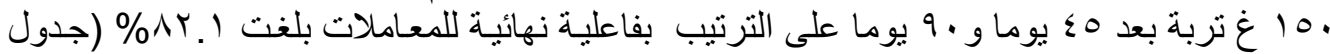

جدول (r) تاثير عملية التشميس واضافة الفطر T. harzianum في كثافة الوحدات التكاثرية

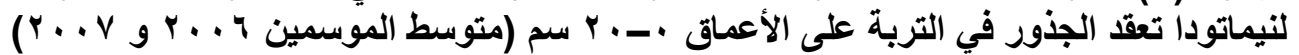

\begin{tabular}{|c|c|c|c|c|}
\hline \multicolumn{4}{|c|}{ متوسط عدد الوحدات التكاثرية النيماتودية في • ه ا غ تربة } & \multirow[b]{2}{*}{ موعد القراعة } \\
\hline \multirow{2}{*}{ بعد ـ يومة من } & \multirow{2}{*}{ من الزراعد هـ يوم } & \multirow{2}{*}{ التشميس } & \multirow{2}{*}{ 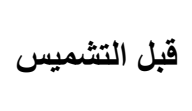 } & \\
\hline & & & & المعاملات \\
\hline AIV.. & VII. & $\Delta \Delta V_{.}$ & $0 \leq Y . \wedge$ & الشـاهد \\
\hline 01.8 & $r v$. & ri.0 & or^.V & التشميس \\
\hline $1 \cdot v . \varepsilon$ & $r r \wedge . q$ & 7.1. & - & T.harzianum الفطر \\
\hline \multirow[t]{3}{*}{11.9} & $\varepsilon .9$ & 19. & $0 \leq Y . \wedge$ & $\begin{array}{c}+ \text { الفطر تثميس } \\
\text { T.harzianum }\end{array}$ \\
\hline & & r^.\& & 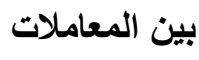 & \multirow{2}{*}{$\mathbf{L S D}_{\mathbf{0 . 0 5}}$} \\
\hline & & rr.q & بين القراعات & \\
\hline
\end{tabular}

وتتفق نتائج هذه الدراسة مع ما توصلت إليه أبحاث سابقة ففي ليبيا أدى تشميس التربة الرطبة

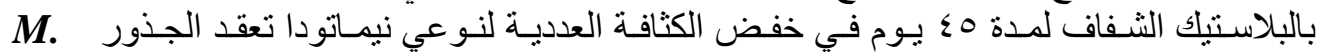
ب. javanica و incognita

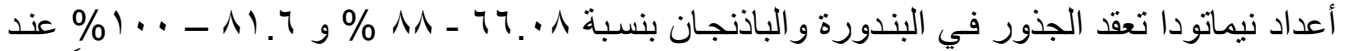

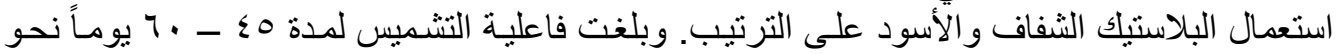

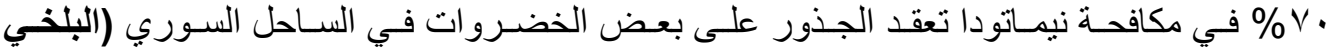

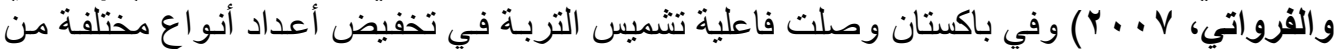

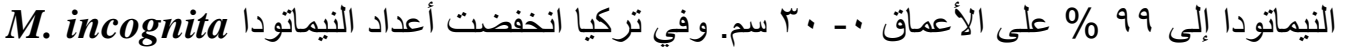

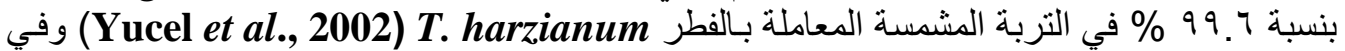

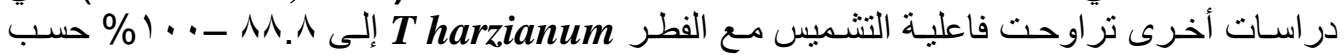
الوقت الذي تحدث فيه عملية التشميس (Yucel et al., 2002).

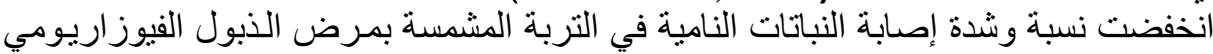

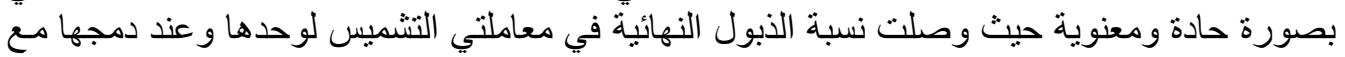

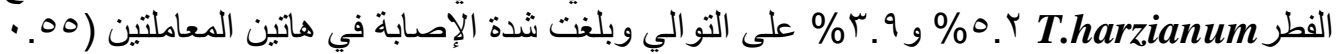

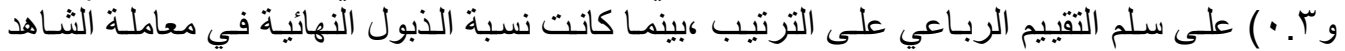

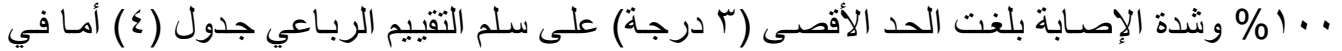

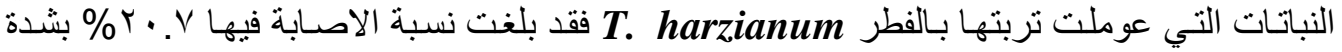
مرضية 0ـ. الني درجة.

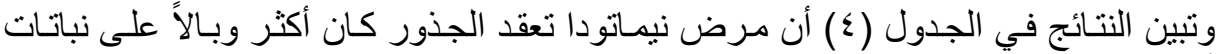

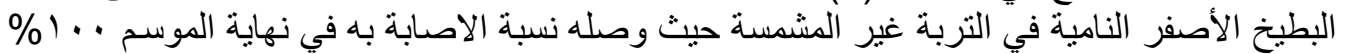

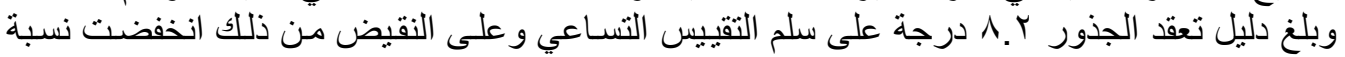

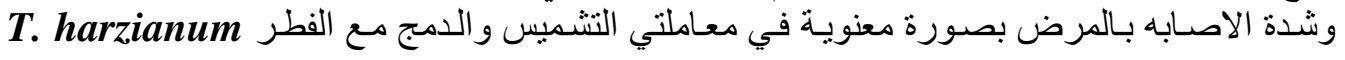

Fayoum J. Agric. Res. \& Dev., Vol.23, No.1, January, 2009 
Tr

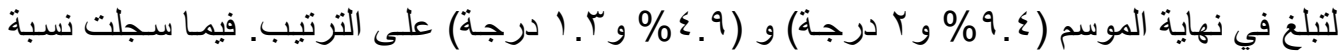

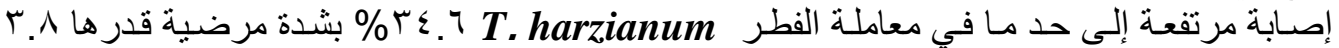
درجة على سلم التقييس التساعي.

جدول ( ) تأثير عملية التشميس وإضـافة الفطر T. harzianum في نسبه وشدة اصـابه البطيخ الأصفر

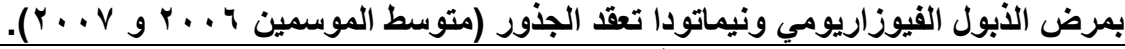

\begin{tabular}{|c|c|c|c|c|}
\hline شعدة الإصابة & 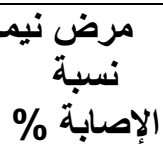 & شيوز (لاريومي & نسبة الأبو & المعاملة \\
\hline r. & 9.8 & .00 & $0 . r$ & التثميس \\
\hline r.^ & $r \varepsilon .7$ & $1 . \leqslant 0$ & $r \cdot . V$ & $\begin{array}{c}\text { Trichoderma الفطر } \\
\text { harzianum }\end{array}$ \\
\hline $1 . r$ & \&.9 & $\cdot r$ & $r . q$ & $\begin{array}{c}\text { Trichoderma } \\
\text { harzianum }\end{array}$ \\
\hline$\Lambda . r$ & $1 \ldots$ & r.. & $1 \ldots$ & الثـاهد \\
\hline 1.1 & $7 . r$ & $\cdot r$ & 0.1 & LSD0.05 \\
\hline
\end{tabular}

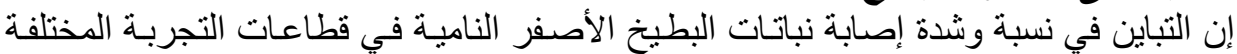

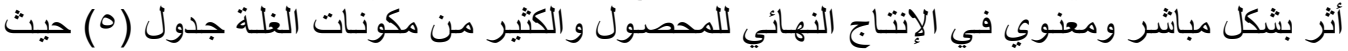

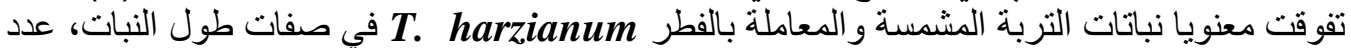

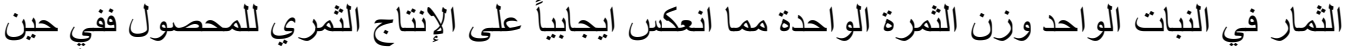

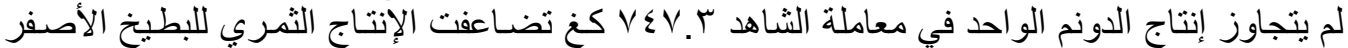

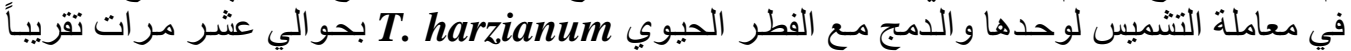

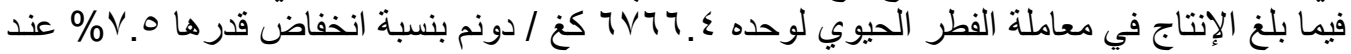

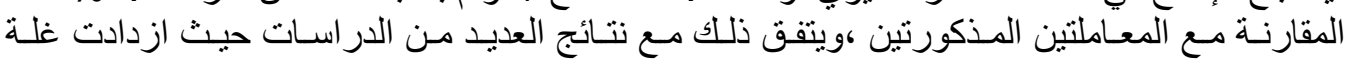

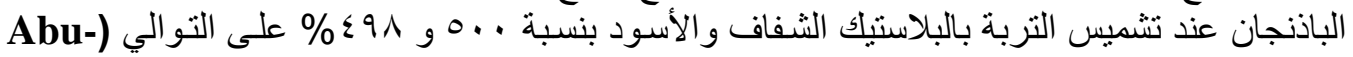

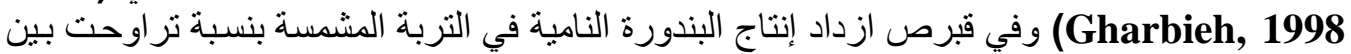

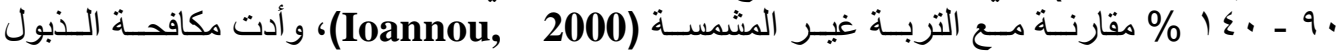

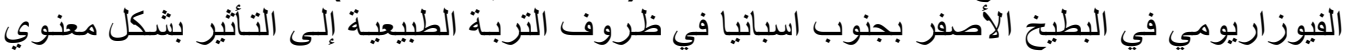
في الإنتاج (Jimenez Diaz et al., 1990) ويفيد الباحث (Gamliel and staplton, 1993)

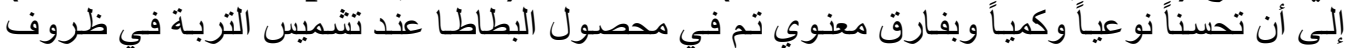

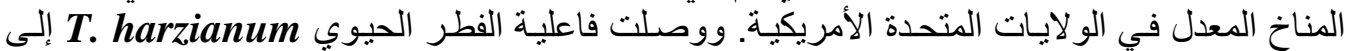

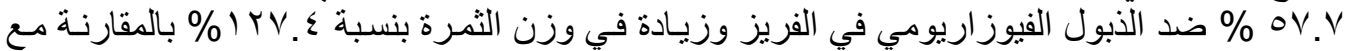

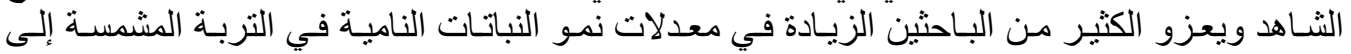

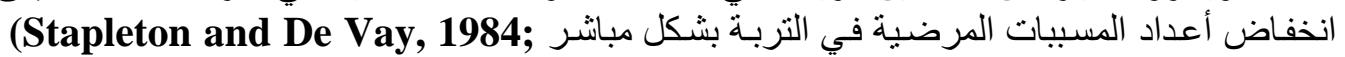
في إلى ازدياد و إتاحة العناصر المعدنية و المو اد العضوية للنباتات بصورة أفضل

في التربة المشمسة al., 1989; Ahmad et al., 1998; Grunzweig et al., 1998).

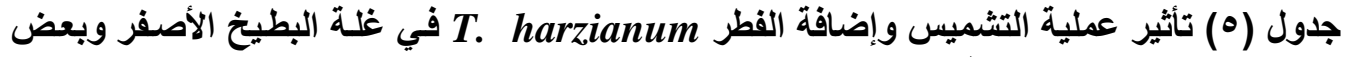

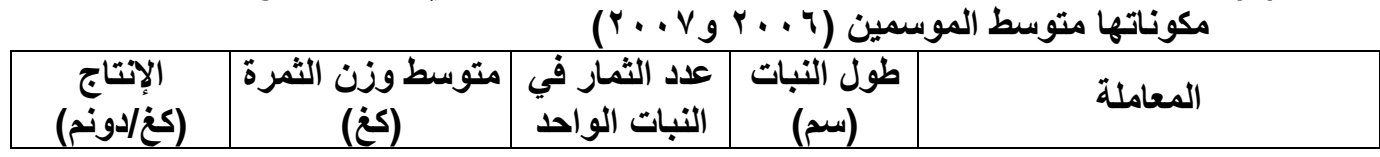

Fayoum J. Agric. Res. \& Dev., Vol.23, No.1, January, 2009 


\begin{tabular}{|c|c|c|c|c|}
\hline$V \leqslant \vee . r$ & $\because 0$ & .7 & VT.V & 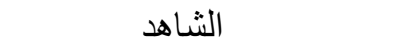 \\
\hline VYOQ.Y & Y.7 & r.o & $177 . r$ & التشميس \\
\hline TVTY. & 1.9 & $r$. & 10V.r & الفطر T. harzianum \\
\hline VYTT.O & $r . \wedge$ & $r . \wedge$ & $1 V \cdot$. & T. harzianum تشميس+الفطر \\
\hline$T T^{\prime} . T^{\prime}$ & .0 & $\because 0$ & 7.1 & $\mathbf{L S D}_{0.05}$ \\
\hline
\end{tabular}

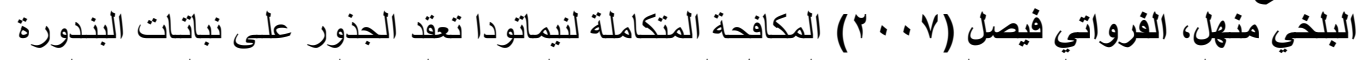

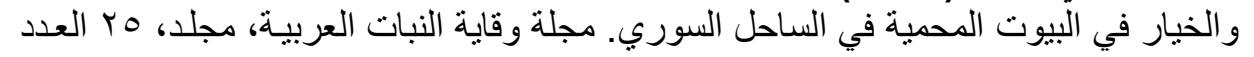

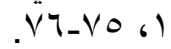

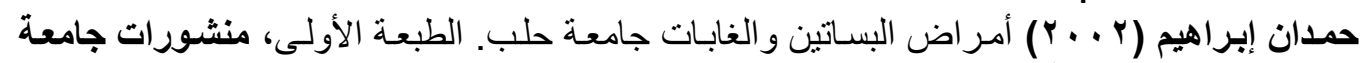

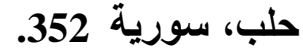

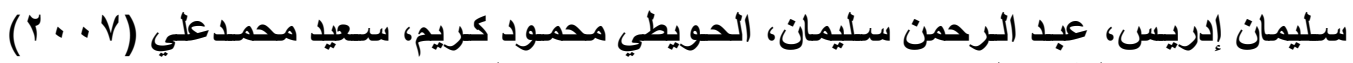

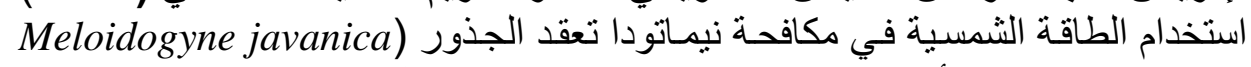

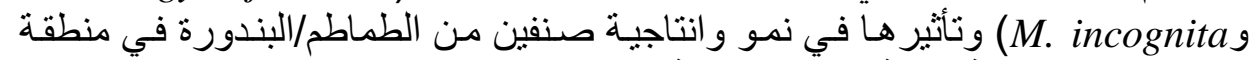

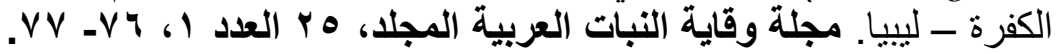

\section{REFERENCES}

Abu-Gharbieh, W.I. (1998) Pre-and post-plant soil solarization. Proc.Sec. Inter. Conf. on Soil Solarization and Integrated Management of Soil Borne Pests.

Aleppo, Syria, 16-21 March, 1997, Published by FAO, 147:15-34.

Ahmad, Y.; A. Hameed and M. Aslam (1998). Soil solarization: A management practice for corn stalk rot, Proc. See. Inter. Conf. on Soil Solarization and Integrated Management of Soil Borne Pest. Aleppo, Syria, 19-21 March, 1997, Published by FAO, 147:141-148.

Chellemi, D.O.; S.M. Olson and D.J. Mitchell (1994). Effects of soil solarization and fumigation on survival of soilborne pathogens of tomato in northern Florida. J. plant Disease, 78: 172-178.

Chen, Y. and J. Katan (1980). Effect of solar heating of soil by transparent polyethylene mulching on their chemical properties. Soil Science, 130: 271-277.

De Vay, J.E. and J. Katan (1991). Mechanisms of pathogen control in solarization soils. CRC press, Boca Raton, F. L., 87-101.

Gamliel, A. and J.J. Stapleton (1993). Effect of chicken compost or ammonium phosphate and solarization on pathogen control, hizosphere microorganisms and lettuce growth. J. Plant Disease, 77: 886-891.

Green, R.J.Jr. (1980). Soil factors affecting survival of microsclerotia of Verticillium dahliae. Phytopathology, 70: 353-355.

Grunzweig, J.M.; J. Katan; Y. Ben-Tal and H.D. Rabinowitch (1998). The role of mineral nutrients in the increased growth response of tomato plants in solarized soil. Plant and Soil 206: 21-27.

Hartz, T. (1993). Solarization is an effective soil disinfestations technique for strawberry production, Hort. Science, 28(2): 104-106.

Fayoum J. Agric. Res. \& Dev., Vol.23, No.1, January, 2009 
ไร

Ioannou, N. (2000). Soil Solarization as asubstitute for Methyl Bromide fumigation in Greenhouse Tomato production in Cyprus. J. Phytoparasitica, 28: 3-12.

Jimenez-Diaz, R.M.; J. Bejarano; M.A. Blanco; J. Gome; R. Gonzalez and J. M. Melero (1990). Control of Verticillium wilt and Fusarium wilt diseases by soil solarization in southern Spain, Abst. of $1^{\text {st }}$ Iner, Conf. on Soil Solarization, 19-25 Feb. Amman, Jordan, P.13.

Katan J. (1981). Solar heating (Solarization) of soil for control of soil born pests. Annual Review of Phytopathology, 19: 211-236.

Katan J.; A. Greenberger; A. Alon and A. Grinstein (1976). Solar heating by polyethylene mulching for the control of diseases caused by soil born pathogens. Phytopathology, 66: 683-688.

Kaewruang, K.; K. Sivasithamparam and G.E. Hardy (1989). Effect of soil solarization with plastic bags on root rot of gerbera (Gerbera jamesonii L.). Plant Soil, 120: 303-306.

Minuto, A.; Q. Migheli and A. Garibaldi (1995). Integrated control of soil-born pathogens by solar heating and antagonistic microorganisms. In: Proceedings of the $4^{\text {th }}$ Int. Symposium on Soil and Substrate Infestation and Disinfestation, Leuven, Belgium (Vanachter, A. (Ed.). Acta Horticulturae, 382: 138-143.

Ristaino, J.B.; K.B. Perry and R.D. Lumsden (1991). Effect of soil solarization and Gliocladium virens on Scierona of Sclerotium rolfsii.soil microbiota and the incidence of southern blight in tomato. Phytopathology, 81: 11171124.

Sarhan, A.R.T. (1990). Control of Fusarium solani in broad bean by solar heating of the soil in northern Iraq. Abstracts of $1^{\text {st }}$ Inter. Conf. on Soil Solarization, 19-25 Feb. Amman, Jordan, P. 14.

Sobh, H. and Y. Abou-jawdah (1998). Effect of soil solarization on soil borne pathogens in Lebanon, Proc. SecInter. Conf. on Soil Solarization and Integrated Management of Soil Born Pests, Aleppo, Syria, 16-21 March, 1997, Published by FAO, 147:149-164.

Stapleton, J.J. and J.E. De Vay (1984). Thermal components of soil solarization as related to changes in soil and root microflora and increased plant growth response. Phytopathol., 74: 255-259.

Stapleton, J.J.; J. Quick and J.E. De Vay (1985). Soil solarization: effect on soil properties, crop fertilization, and plant growth. Soil Biol. and Biochem., 17: 369-373.

Stapleton, J.J. (2000). Soil solarization in various agricultural production systems. Crop Protection, 19: 837-841.

Yucel, S.; H. Elekciolu; A. Uluda; C. Can; U. Gozel; M.A. Sout; A. Ozarslandan and E. Aksoy (2002). The Second year results of Methyl bromide alternatives in the eastern Mediterranean part of Turkey. In: Proceedings of the Annual International Research Conference on Methyl Bromide Alternatives and Emissions Reductions, Florida, pp. 10-1/10-4.

Fayoum J. Agric. Res. \& Dev., Vol.23, No.1, January, 2009 
70

\title{
EFFECTIVENESS OF SOIL SOLARIZATION AND Trichoderma harzianum IN THE CONTROL OF FUSARIUM WILT DISEASE AND ROOT-KNOT NEMATODE ON MELON UNDER DEIR EZZOR CONDITIONS, SYRIA
}

H. Al-Dakheel and F. Madhour

Dept. Plant Protection, Faculty of Agric., Al-Furat University

\begin{abstract}
The effectiveness of soil solarization and or fungi biological control (Trichoderma harzianum) against fusarium wilt and root-knot nematode diseases in musk melon was tested. This research has been implemented under field conditions in Dier Ezzor governorate in the east side of Syria. Results showed an increase in temperature at the depth $5-20 \mathrm{~cm}$ after 60 days of covering with transparent plastic grading between $12.3-19.3^{\circ} \mathrm{C}$. The effectiveness of soil solarization alone or when incorporated with fungi (Trichoderma harzianum) reached $99 \%$ for fusarium fungi populations. The solarization inhibited fusarium fungi successfully preventing pathogen population recovery for 90 days after planting; where the activity reached 96.4\%. However, no fungi was found at the same time by incorporation solarization with Trichoderma harzianum. The general average of reproductive unit of the root-knot nematode was affected in the solarized soil at the same prior time and depth where activity reached $96 \%$. The rate of injury of plants with fusarium wilt diseases decreased in solarized soil or in sectors of treatment in corporation with fungi significantly and eventually and did not surpassed 5.2$3.9 \%$, respectively, compared to control $(100 \%)$ The rate of injury the root-knot nematode diseases decreased in the same treatments to 4.9-9.4\% compared to $100 \%$ in control. The solarization and fungi treatment increased the characteristics and final yield of musk melon yield.
\end{abstract}

Key words: Soil solarization, T. harzianum, Root -knot nematode, Fusarium wilt, Melon.

Fayoum J. Agric. Res. \& Dev., Vol.23, No.1, January, 2009 